\title{
Acoustic Behaviour of Nonwovens
}

\author{
N Gokarneshan ${ }^{1 *}$, V Krishna Kumar ${ }^{2}$ \\ ${ }^{1}$ SNS Rajalakshmi College of arts and science, India \\ ${ }^{2}$ SMK Fomra Institute of technology, India
}

*Corresponding author: N Gokarneshan, Department of costume design and fashion, SNS Rajalakshmi College of arts and science, Coimbatore, India.

Received Date: June 05, 2020

Published Date: June 17, 2020

\begin{abstract}
The article reviews the acoustic properties of nonwoven fabrics. sound absorption coefficients (SACs) of needle-punched and thermal-bonded nonwovens produced from polyester (PET) fibers with various cross sections, i.e., hollow, round and hexaflower, blended with a low melt PET, was reported. Efforts have been taken to develop relatively lightweight and fibrous acoustic webs. Nonwoven webs that contain bicomponent filaments with islands-in-the-sea cross sections were produced by spun bonding, which involves the extrusion of sea and island polymer melts through dies, cooling and attenuating the bicomponent filaments by high-velocity air streams. Nylon 6 and polyethylene were used as the island and sea polymers, respectively.

Keywords: Nonwoven; PET fiber; Sound absorption; Island in the sea, Spun bond; Cross section; Taguchi
\end{abstract}

\section{Introduction}

As noise problems can result in many issues like hearing loss, decreased work efficiency, fatigue, and even psychophysiological problems, it is important to control noise in automobiles or other vehicles, industrial machines and constructions. Acoustic materials like sound absorbers, sound barriers and silencers can enable noise control to be achieved, through redesign of sound sources and hearing protection aids like earplugs [1]. The most preferred textile based sound absorbers for noise control applications are nonwoven materials because of their high porosity and low solid volume fraction (SVF). Most nonwovens would have a SVF of $20 \%$ or less [2]. Nonwovens are complex fibrous and porous structures, and often have very low solidity. They are inherently lightweight due to pores filled with air. The pores are also in the form of a bundle of capillaries. Nonwovens are known to have useful acoustical properties and are used as sound absorbers because of their complex fiber network geometry, bulk and low density $[3,4]$. These structures inherently have interconnected pores with tortuous paths. Porous sound absorbers are widely used to reduce noise and to control reverberation time.

\section{Influence of Fibre Cross Section and Various} Bonding Techniques Adopting Taguchi

Owing to their high porosity and low solid volume fraction nonwoven materials are considered to be the most appropriate textile based sound absorbers for noise control applications. The solid volume fraction in majority of nonwovens could be $20 \%$ or less. The structure of nonwovens inherently has pores with tortuous paths, allowing the sound waves to enter through them. Various kinds of pores like 'open' or 'closed' can be present in a porous material [6]. 'Open' pored materials have a continuous channel of communication with the external surface of the body. The open-pored structure of nonwovens provides a proper medium for sound waves to be absorbed by three mechanisms which are: converting the sound energy into heat in the material, viscous losses because of the oscillating air flow entering the porous areas inside the material and vibration of fibers due to vibration of air particles caused by the sound waves [7]. Since nonwoven materials have a complex structure, there are many variables influencing 
their sound absorption and insulation properties. Previous studies investigated especially the effect of fiber denier and/or fiber cross section, areal density (gsm), air permeability and production machine parameters/production methods on acoustic performance [8-11]. There have been reports relating to the indluences of fiber denier, fiber shape and fabric density on compression molded vertically lapped fabrics. Fabrics made from 3 denier fibers acted as better sound insulators than those made from 15 denier fibers for three different fiber shapes (round, trilobal, and 4DG), and the vertically lapped nonwoven fabrics made from 4DG and trilobal fibers had better sound insulation results than those made from round fibers. Studies have been made from polypropylene fibers and it is reported that sound transmission loss of the samples was most influenced by the basis weight of initial carded web, and it was increased by increasing the web mass. In addition, the frequency, needle penetration depth and needle punch density variables were found as effective on sound transmission loss. Whereas another work reported on needled nonwoven samples produced from blends of milkweed/polypropylene fibers in different proportions [12]. Fiber blend ratio, punch density and areal density were selected as predictor variables. As a result, blend ratio and areal density were reported as effective variables, while punch density had insignificant effect on acoustic performance. It has been reported that there was a direct relationship between areal density and sound absorption efficiency. It is also found that samples produced with high percentage of hollow fibers showed the highest rate of sound absorption. Studied on Struto nonwovens with varying thicknesses and gsm values have been done.

The results indicated that fabric thickness, fiber fineness and fabric gsm had a significant positive effect on the sound absorption performance of Struto nonwovens. It is also reported that the fiber fineness and fabric gsm were very important factors affecting the specific airflow resistance of the sound absorber, so that higher airflow resistance led to better sound absorption values of Struto nonwovens. It is reported that nonwoven samples developed by using microfibers and produced with lower areal density and higher thickness resulted in better sound absorption values. The bonding method, i.e., water-jet bonding or needle punching, of polyester based nonwovens did not significantly affect the sound absorption characteristics of the material. It is reported that air-laid samples showed better acoustic performance than carded samples because more random lay of fibers resulted in more tortuous structure and smaller pores which yielded better sound absorption [13]. In addition, no significant difference was observed between needled and needled-thermal bonded samples in terms of sound absorption property. In this study, the acoustic performance of nonwoven samples produced from polyester (PET) fibers with various cross sections such as hollow, round and hexaflower (with hexagonal petal shape cross section and hollow cavity) blended with low melt PET (LMPET) was reported. The fiber cross section providing higher surface area can improve the sound absorption performance of acoustic nonwoven materials since higher total fiber surface area increases the possibility for a sound wave to interact with the fibers within the material. However, the number of studies investigating the effect of fiber cross section on acoustic performance is limited in the literature. In addition, to the best of knowledge, the study is the first in the literature that shows the acoustic performance of a fiber with both hexagonal petal shape cross sectional and hollow cavity. This fiber, which is developed for sound absorption applications, provides high resilience and bulkiness properties [14]. Moreover, the sound absorption property of needle punch bonded and thermal bonded samples were compared in the current study, which was rarely studied. In addition, different from the previous studies, statistical analyses were performed not only on an average sound absorption coefficient but also on sound absorption coefficients at different sound frequencies (i.e. 500, 2000, and $4000 \mathrm{~Hz}$ ). This study aimed to investigate whether the effect of production parameters change based on frequency levels, and determine the most effective production parameters on sound absorption of the produced nonwovens. Therefore, the current study contributes to the literature by analysing the effects of different bonding methods and fiber cross section on sound absorption properties of nonwovens at different sound frequencies using Taguchi experimental design approach. Based on the Taguchi method, the optimum levels for each production parameter were obtained to produce a nonwoven sample with the highest sound absorption coefficient (SAC) value at a mid-frequency of $2000 \mathrm{~Hz}$, which is used to evaluate sound absorbing materials for automotive interior [15]. In addition, a comparison of sound absorption properties of produced nonwoven samples with a commercial acoustic material was performed to evaluate the potential use of produced nonwovens.

In the investigation considered, the effect of production factors of nonwoven samples like web bonding, cross section of fiber, blend ratio of PET/LMPET, areal density and punch density on the sound absorption coefficient has been analysed with Taguchi experimental design approach. According to Taguchi L18 OA, the analysis showed that web bonding had the most significant effect on SAC at $500 \mathrm{~Hz}$ frequency because web bonding method directly affected sample thickness and thicker materials showed better performance at low frequencies. On the other hand, at $4000 \mathrm{~Hz}$, the analysis showed that areal density was the most effective variable on SAC values, whereas web bonding method was the least effective variable on SAC values.

According to the analysis at a mid-frequency of $2000 \mathrm{~Hz}$, both areal density and web bonding method were statistically significant variables, whereas blend ratio of PET/ LMPET and fiber cross section were statistically insignificant variables for sound absorption performance of the nonwoven samples. Production parameters in terms of their effect on SAC were ranked as areal density, web bonding, blend ratio and cross section of fibers. According to Taguchi L9 OA experimental design, punch density had even a lower effect than cross section of fibers on SAC value. 
Owing to its larger surface area having hexagonal petal shape cross section the acoustic performance showed a positive trend in the case of hexaflower PET fiber in nonwoven samples in comparison with hollow and round PET fibers. Moreover, SAC values were obtained between 0.15 and 0.36 for needle punch bonded samples; whereas thermal bonded samples showed SAC values between 0.25 and 0.50 at $2000 \mathrm{~Hz}$ which confirmed better acoustic performance of thermal bonded samples. In addition, it was found that increasing the areal density from 300 to $650 \mathrm{~g} /$ $\mathrm{m} 2$ improved the sound absorption performance of the nonwoven samples. As a result, areal density was found to be the most influential parameter on sound absorption coefficient at a midfrequency of $2000 \mathrm{~Hz}$. Increasing the weight of PET fiber ratio from $50 \%$ to $65 \%$ in the PET/LMPET blend affected the acoustic performance positively, whereas increasing the weight of PET fiber ratio from $65 \%$ to $80 \%$ did not lead to a significant change in the $\mathrm{S} / \mathrm{N}$ ratio because bonding of the material may not significantly change the bulkiness of the material when the LMPET fiber ratio reduced below 35\% [16]. Optimum production levels in OA L18 design were obtained as 'thermal, hexaflower PET, 80/20 PET/ LMPET and $650 \mathrm{~g} / \mathrm{m} 2$ '. SAC value of the sample with the optimum production levels was calculated with Minitab software as 0.57 at $2000 \mathrm{~Hz}$. When a comparison was performed with a commercial sound absorption material, this sample showed the same sound absorption performance with the commercial material having a higher areal density of $1000 \mathrm{~g} / \mathrm{m} 2$. As a result, the sample produced based on optimum production levels with a SAC value of 0.57 at $2000 \mathrm{~Hz}$ may have a potential use in acoustic applications in the automotive industry.

\section{Effect of Sea Polymer Removal in Islands in the Sea Spun Bonded Non-Woven}

A number of studies have been carried out in sound absorption related nonwovens for assessment of the influences of structural factors [17-21]. The frictional resistance between fibres causes absorption of sound in nonwovens. During sound wave propagation the acoustic energy gets dissipated by the air trapped in the pores. In bulky and thick nonwovens, sound absorption is generally higher due to longer tortuous paths that offer more frictional resistance while sound waves pass through. The structures having higher numbers of fibers per unit volume and a large fiber surface area also offers more frictional resistance, which leads to higher sound absorption performance. Fiber shape is another important parameter, since there is the possibility to increase the total fiber surface area. Sound waves interact with a larger solid surface during propagation that increases sound absorption.

It is necessary to use thinner fibres in order to form structures having more fibers per unit volume. Sub micro and micro fibre based nonwovens can be produced by the spun bonding process with bicomponent filament technology combined with a fibersplitting process. Segmented-pie or islands-in-the-sea cross sections are often chosen to produce bicomponent filaments. Fiber diameters ranging from $0.3-5 \mathrm{~mm}$ can be obtained after a suitable post-process that releases the finer fibers $[22,23]$. Producing thin fibers with islands-in the-sea cross sections is feasible since the number of islands in a circular bicomponent filament is limitless theoretically and the sea component that surrounds island fibers makes the whole bicomponent filament more spinnable [24]. Island fibers of the resulting nonwoven can be split by mechanical forces. For instance, high-speed hydro entangling water jets can be used for this purpose. It has been demonstrated that hydro entangled islands-in-the-sea spun bond structures can form superior structures in terms of strength, durability and porosity [25]. It was also shown that islands-in-the-sea fibers can be fibrillated/fractured by hydro entangling to deliver micro- and Nano denier fibers with tremendously high surface areas. When considering end uses such as filtration and sound absorption, fibrous structures with high fiber surface areas appear prospective. Recently, investigation has been done on the aerosol filtration characteristics of these structures and found that the hydro entangling process improved the aerosol filtering efficiency by increasing the electrostatic charging on the web [26]. Acoustical absorptive properties of hydro entangled spun bonded nonwovens made from islands-in-the-sea bicomponent filaments were reported. Nonwovens produced from higher numbers of island fibers absorbed more sound energy according to the results. Another method that can release island fibers from bicomponent filaments is the dissolution of the sea component. After removing the sea polymer from the bicomponent filament by dissolving, nonwovens only contain thin island fibers, have higher total fiber surface areas and also become lighter. A dissolution technique has been used for the spun bonded webs and exploration done regarding the idea that the islands-in-the-sea configuration is a promising technique for high-speed and high-throughput production of strong and lightweight nonwovens comprised of micro and nanofibers. Most of the studies on islands-in-the-sea webs have primarily focused on optimizing their mechanical and bonding strengths, and the formation of durable nonwoven fabrics $[27,28]$. The aim of this study is to form lightweight and thin fibrous structures by removing the sea polymer and investigate the sound absorption performance of the islands-in-the-sea nonwovens with varying numbers of island fibers.

The process of dissolution highly enables the elimination of the sea polymer from spun bonded nonwovens which comprise of bicomponent filaments with islands in-the-sea cross sections. After removing the sea polymer, spun bonded nonwovens contain only thin island fibers and also get lighter. Sea polymer removal leads to further bicomponent filament fibrillation, especially for the spun bonded webs processed at lower hydro entangling energies (one and two passes) [29]. Island fibers released due to fibrillation have a positive influence on sound absorption behaviour. It was also shown that sound absorption coefficients of the dissolved structures increase by the number of island fibers. Findings indicate 
that dissolution method given in this study can be used to form lightweight and fibrous sound absorptive spun bonded nonwovens. These structures have the potential to be used in cars in place of high volume, fibrous bulky sound absorbers.

\section{Conclusion}

An innovative research has been carried out on the acoustic performance of a hexaflower PET fiber. The carded fibres have been made into two sets of nonwovens by adoption of needle punching as well as thermal bonding in air. Another set of nonwoven has been produced by needling at different punch densities. Design of experiments was planned according to Taguchi method. Relationship between production parameters and SAC was analysed using Minitab software. The most important independent variables affecting the sound absorption were areal density and web bonding method. The sample produced according to optimum production levels reached to a SAC value of 0.57 at $2000 \mathrm{~Hz}$ which could be a suitable choice for acoustic applications in the automotive industry. Nylon 6 and polyethylene were used as the island and sea polymers, respectively. Webs were hydro entangled with high-pressure water jets prior to the dissolving process to obtain fiber entanglement. Sea polymer was removed from the spun bonded nonwovens by using a reflux dissolution setup. Weight, thickness, air permeability, pore size and sound absorption coefficients of the nonwoven samples were measured before and after the sea polymer removal. Results demonstrated that sea polymer removal led to further bicomponent filament fibrillation, which affected sound absorption positively. The structure with the higher number of island fibers had better acoustical properties. Lightweight and fibrous acoustic nonwovens can be obtained with the method given in this study.

\section{Acknowledgement}

None.

\section{Conflict of Interest}

No conflict of interest.

\section{References}

1. Nayak R, Padhye R (edts) (2016) Acoustic textiles. Springer, Singapore.

2. Suvari F, Ulcay Y, Maze B, Pourdeyhimi B (2013) Acoustical absorptive properties of spunbonded nonwovens made from islands-in-the-sea bicomponent filaments. Journal of the Textile Institute 104(4): 438-445.

3. Barron RF (2003) Industrial noise control and acoustics. New York: Marcel Dekker, USA.

4. Arenas JP, Crocker M (2010) Recent trends in porous sound absorbing materials. Sound and Vibration 44(7): 12-17.

5. Chen CY (2016) Textile advances in the automotive industry. In Shishoo R (edts.) Acoustic Textiles New York: Woodhead Publishing, USA, 198228.

6. Ramamoorthy M, Rengasamy RS (2018) Study on the effects of denier and shapes of polyester fibres on acoustic performance of needlepunched nonwovens with air-gap: Comparison of artificial neural network and regression modelling approaches to predict the sound absorption coefficient of nonwovens. Journal of the Textile Institute 110(5): 715-723.

7. Tascan M, Vaughn EA(2008) Effects of fiber denier, fiber cross-sectional shape and fabric density on acoustical behavior of vertically lapped nonwoven fabrics. Journal of Engineered Fibers and Fabrics 3(2).
8. Ghorbani K, Hasani H, Zarrebini M, Saghafi R (2016) An investigation into sound transmission loss by polypropylene needle punched nonwovens. Alexandria Engineering Journal 55(2): 907-914.

9. Küçük M, Korkmaz Y (2012) The effect of physical parameters on sound absorption properties of natural fiber mixed nonwoven composites. Textile Research Journal 82(20): 2043-2053.

10. Mahmoud AA, Ibrahim GE, Mahmoud ER (2011) Using nonwoven hollow fibers to improve cars interior acoustic properties. Research Journal of Textile and Apparel 8(1): 49-56.

11. Yang T, Xiong X, Mishra R, Novák J, Militký J (2016) Acoustic evaluation of struto nonwovens and their relationship with thermal properties. Textile Research Journal 88(4): 426-437.

12. Hassanzadeh S, Zarrebini M, Hasani H (2014) An investigation into acoustic properties of lightly needled estabragh nonwovens using the taguchi method. Journal of Engineered Fibers and Fabrics 9(3).

13. Jayaraman K (2005) Acoustical absorptive properties of nonwovens. Raleigh, NC: North California State University, USA.

14. Huvis (2018) Hexaflower: A heterogeneous hollow fiber with excellent sound absorption and insulation.

15. Liu Y, Jia YB, Zhang XJ, Liu ZC, Ren YC, et al. (2013) Noise test and analysis of automobile engine. Applied Mechanics and Materials 307: 196-199.

16. Handan P, Kayaoğlu BK (2019) Analysis of the effect of fiber cross section and different bonding methods on sound absorption performance of PET fiber based nonwovens using Taguchi method, The Journal of The Textile Institute 111(20): 575-585.

17. Aso S, Kinoshita R (1964) Sound absorption characteristics of fiber assemblies. J Text Mach Soc Japan 10: 209-217.

18. Genis AV, Kostyleva EY, Kostylev VA (1990) Sound absorbing properties of fibrous materials prepared by the aerodynamic method. Fibre Chem 21: 389-392.

19. Suvari F, Ulcay Y, Pourdeyhimi B (2016) Sound absorption analysis of thermally bonded high-loft nonwovens. Text Res J 86(8): 837-847.

20. Russell SJ (2007) Handbook of nonwovens. Cambridge: Woodhead Publishing Limited, UK.

21. Aso S, Kinoshita R (1963 Absorption of sound wave by fabrics. J Text Mach Soc Japan 9: 32-39.

22. Fedorova N, Pourdeyhimi B (2007) High strength nylon micro- and nanofiber based nonwovens via spunbonding. J ApplPolymSci 104(5): 3434-3442.

23. Hollowell KB, Anantharamaiah N, Pourdeyhimi B (2013) Hybrid mixed media nonwovens composed of macrofibers and microfibers. Part I: Three-layer segmented pie configuration. J Text Inst 104(9): 972-979.

24. Anantharamaiah N, Verenich S, Pourdeyhimi B (2008) Durable nonwoven fabrics via fracturing bicomponent islands-in-the-sea filaments. J EngFabr Fibers 3(3): 1-9.

25. Durany A, Anantharamaiah N, Pourdeyhimi B (2009) High surface area nonwovens via fibrillating spun bonded nonwovens comprising islands-in-the-sea bicomponent filaments: Structure-process-property relationships. J Mater Sci 44: 5926-5934.

26. Yeom BY, Pourdeyhimi B (2011) Aerosol filtration properties of PA6/ PE islands-in-the-sea bicomponent spunbond web fibrillated by highpressure water jets. J Mater Sci 46: 5761-5767.

27. Fedorova N, Verenich S, Pourdeyhimi B (2007) Strength optimization of thermally bonded spunbond nonwovens. J Eng Fiber Fabr 2(1): 38-48.

28. Pourdeyhimi B, Fedorova NV, Sharp SR (2006) Lightweight hightensile, high-tear strength bicomponent nonwoven fabrics. Patent US20060223405A1, USA.

29. Fatih S, Yusuf U, Behnam P (2018) Influence of sea polymer removal on sound absorption behavior of islands-in-the-sea spunbonded nonwovens, Textile Research Journal 89(12): 2444-2455. 